\title{
Is Human Photosynthesis a Fact?
}

\section{Oktar $\mathbf{N}^{*}$}

Ege University, Department of Neurosurgery, Izmir, Turkey

Melanin is a pigment and widespread in nature, being found in most organisms. In humans, animal pigment is that the primary determinant of colouring. It's conjointly found in hair, pigmented tissue underlying the iris of the attention, and also the stria vascularis of the sense organ. Within the brain, tissues with animal pigment embrace the medulla and pigment-bearing neurons inside areas of the brain-stem, like the locus coeruleus and also the neural structure and body structure reticularis of the endocrine gland.

Eumelanin polymers have long been thought to comprise various cross-linked five,6-dihydroxyindole (DHI) and five,6-dihydroxyindole2 -carboxylic acid (DHICA) polymers. However, recent analysis into the electrical properties of eumelanin has indicated that it should encompass additional basic oligomers adhering to at least one another by another mechanism [1-5].

Neuromelanin is that the dark pigment gift in pigment-bearing neurons of chiefly four deep brain nuclei. These square measure the neural structure - Pars Compacta half, the locus coeruleus, the dorsal motor nucleus of the nervus vagus (cranial nerve $\mathrm{X}$ ), and therefore the median ridge nucleus of the neural structure. each the neural structure and locus coeruleus are often simply known grossly at the time of autopsy thanks to their dark pigmentation. In humans, these nuclei aren't pigmented at the time of birth, however develop pigmentation throughout maturation to adulthood. neural structure animal pigment acts as semiconductor, transmission and modulating nervous impulses, in a very reversible method. In fact, neural structure animal pigment is absent or considerably scarce in 2 conditions of life during which the coordination of movement is either inefficient (newborn babies) or powerfully compromised (Parkinson Disease). to ascertain this assumption, any investigation of nucleus caudatus, putamen, pallidum, neural structure pars compacta and reticulata, nucleus hypothalamicus is usually recommended [6].

Recent analysis by Sulzer et al. suggests that animal pigment could serve a protecting role aside from photoprotection [7] animal pigment is ready to effectively ligate metal ions through its treat and phenoplast radical teams, in several cases rather more with efficiency than the powerful chelating substance ethylenediaminetetraacetate (EDTA). Thus, it's going to serve to sequester probably toxicant metal ions, protective the remainder of the cell. This hypothesis is supported by the very fact that the loss of neuromelanin discovered in degenerative disorder is in the course of a rise in iron levels within the brain.

Although the practical nature of neuromelanin is unknown within the brain, the pigment is created from oxyradical metabolites of aminoalkane neurotransmitters together with monoamine neurotransmitter and vasoconstrictor. Luigi Zecca associate degreed David Sulzer gift proof that neuromelanin pigment is an autophagy product that accumulates in lysosomes, that are unable to effectively degrade it [5,7-9]. During this method, the synthesis of neuromelanin is protecting as its encapsulation within the autophagic organ removes it from reacting with sites within the vegetative cell cytoplasm that might result in neurotoxicity. Alternative evidences, suggests an additional active role $[1,2,10]$.
On the opposite hand, animal pigment precursors (e.g. DHI) deserves attention having sturdy antioxidative result on retinal cells and a few toxicity on mouse malignant melanoma cells in vitro and in vivo [3].

Herrara AS and his colleagues dealing regarding what they referred to as as 'Human Photosynthesis', water molecule associated animal pigment square measure underneath scientific scope as chief actor \& player of an unknown pic [4].

Although a number of the steps in chemical change square measure still not utterly understood, the chemical process equation has been well-known since the nineteenth century.

A 2010 study by researchers at Tel Aviv University in Israel discovered that the oriental vespid wasp (Vespa orientalis) converts daylight into power employing a pigment referred to as xanthoperin. This is often the primary scientific proof of a member of the kingdom Animalia participating in chemical change [8].

\section{References}

1. Double KL, Dedov VN, Fedorow H, Kettle E, Halliday GM, et al. (2008) The comparative biology of neuromelanin and lipofuscin in the human brain. Cell Mol Life Sci 65: 1669-1682.

2. Elstner M, Müller SK, Leidolt L, Laub C, Krieg L, et al. (2011) Neuromelanin neurotransmitter status and brainstem location determine the differentia vulnerability of catecholaminergic neurons to mitochondrial DNA deletions. Mol Brain 4: 43.

3. Heiduschka P, Blitgen-Heinecke P, Tura A, Kokkinou D, Julien S, et al. (2007) Melanin Precursor 5,6-Dihydroxyindol: Protective Effects and Cytotoxicity on Retinal Cells in vitro and in vivo. Toxicol Pathol 35: 1030-1037.

4. Herrera AS, Esparza MCA, Arias RIS, Arias PES, Arias MPS (2012) Human Photosynthesis and Central Nervous System's Diseases J Neurol Sci Turk 29: 654-668.

5. Liu Y, Hong L, Kempf VR, Wakamatsu K, Ito S, et al. (2004) lon-exchange and adsorption of Fe(III) by Sepia melanin. Pigment Cell Research 17: 262-269.

6. Nicolaus BJ (2005) A critical review of the function of neuromelanin and an attemp to provide a unified theory. Med Hypotheses 654: 91-96.

7. Sulzer D, Mosharov E, Talloczy Z, Zucca FA, Simon JD, et al. (2008) Neuronal pigmented autophagic vacuoles: Lipofuscin, neuromelanin, and ceroid as macroautophagic responses during aging and disease. Journal of Neurochemistry 106: 24-36.

8. Plotkin MHI, Hod I, Zaban A, Boden SA, Bagnall DM, et al. (2010) Solar energy harvesting in the epicuticle of the oriental homet (Vespa orientalis). Naturwissenschaften 97: 1067-1076.

*Corresponding author: Nezih Oktar, Ege University, Department of Neurosurgery, Izmir, Turks \& Caicos Islands, E-mail: nezih.oktar@ege.edu.tr

Received July 02, 2013; Accepted August 16, 2013; Published August 19, 2013

Citation: Oktar N (2013) Is Human Photosynthesis a Fact? J Bioanal Biomed 5 077-078. doi:10.4172/1948-593X.1000085

Copyright: (c) 2013 Oktar N. This is an open-access article distributed under the terms of the Creative Commons Attribution License, which permits unrestricted use, distribution, and reproduction in any medium, provided the original author and source are credited. 
9. Oktar N (2012) Neuromelanin and Human Photosynthesis. J Neurol Sci Turk 29: $442-443$

10. Zecca L, Casella L, Albertini A, Bellei C, Zucca FA, et al. (2008) Neuromelanin can protect against iron-mediated oxidative damage in system modeling iron overload of brain aging and Parkinson's disease. J Neurochem 106: 18661875. 